\title{
Quelques enseignes de pèlerins et des moules de production de petits objets en plomb découverts à Rennes
}

Some Pilgrim Badges and Production Moulds for Small Lead Objects Discovered in Rennes

\section{Françoise Labaune-Jean}

\section{(2) OpenEdition \\ Journals}

\section{Édition électronique}

URL : http://journals.openedition.org/abpo/2858

DOI : $10.4000 / a b p o .2858$

ISBN : 978-2-7535-3977-8

ISSN : 2108-6443

\section{Éditeur}

Presses universitaires de Rennes

\section{Édition imprimée}

Date de publication : 15 décembre 2014

Pagination : 7-22

ISBN : 978-2-7535-3975-4

ISSN : 0399-0826

Référence électronique

Françoise Labaune-Jean, "Quelques enseignes de pèlerins et des moules de production de petits objets en plomb découverts à Rennes ", Annales de Bretagne et des Pays de l'Ouest [En ligne], 121-4 | 2014, mis en ligne le 15 décembre 2016, consulté le 19 avril 2019. URL : http:// journals.openedition.org/abpo/2858; DOI : 10.4000/abpo.2858 


\title{
Quelques enseignes de pèlerins et des moules de production de petits objets en plomb découverts à Rennes ${ }^{1}$
}

\author{
Françoise LABAUNE-JEAN \\ avec la collaboration de Denis BRUNA \\ INRAP Grand Ouest (Rennes), UMR 6566
}

Dans le cadre de l'installation du métro-VAL à Rennes, une fouille archéologique s'est déroulée en 1998 sur l'emprise de la station Sainte-Anne. Cette opération a permis la mise au jour d'un ensemble de structures rattachées à l'occupation médiévale et moderne du secteur.

\section{Le contexte historique}

Après l'édification de la seconde enceinte de Rennes² ${ }^{2}$, de 1421 à 1448, le faubourg se situe à proximité de la barbacane la plus septentrionale de la fortification. C'est dans le contexte d'essor urbain de la capitale bretonne au début du XIv ${ }^{\mathrm{e}}$ siècle $^{3}$, qu'est fondé en 1340 l'hôpital Sainte-Anne ${ }^{4}$ sous l'impulsion de dix confréries ouvrières de Rennes. C'est également l'époque des crises démographiques provoquées par les épidémies et les famines précédant la Peste noire, qui touche la Bretagne en 1348. Cet hôpital comprend principalement une salle des malades, une chapelle et des bâtiments annexes situés à l'intérieur d'un enclos (figure 1). C'est une partie des vestiges de ces derniers qui fut appréhendée lors des fouilles de la station de la première ligne de métro.

1. Nous tenons à remercier Denis Bruna, pour sa collaboration et sa contribution dans l'identification des enseignes de pèlerinage.

2. Dubant, Didier, "Les enceintes de Rennes ", dans " La Bretagne ducale ", Histoire Médiévale, hors série ${ }^{\circ} 3$, août-octobre 2003, p. 48-53.

3. Arch. mun. de Rennes, liasse 327. Acte de fondation de l'hôpital Sainte-Anne, décembre 1340.

4. L'église Saint-Aubin voisine de l'hôpital est mentionnée dès 1158 comme faisant partie des faubourgs de la cité (ecclesia sancti albini in suburbio constitua). Arch. dép. d'llle-et-Vilaine, 1F501/1, copie du cartulaire de Saint-Melaine, $\mathrm{f}^{\circ} 32$, acte ${ }^{\circ} 22$. 


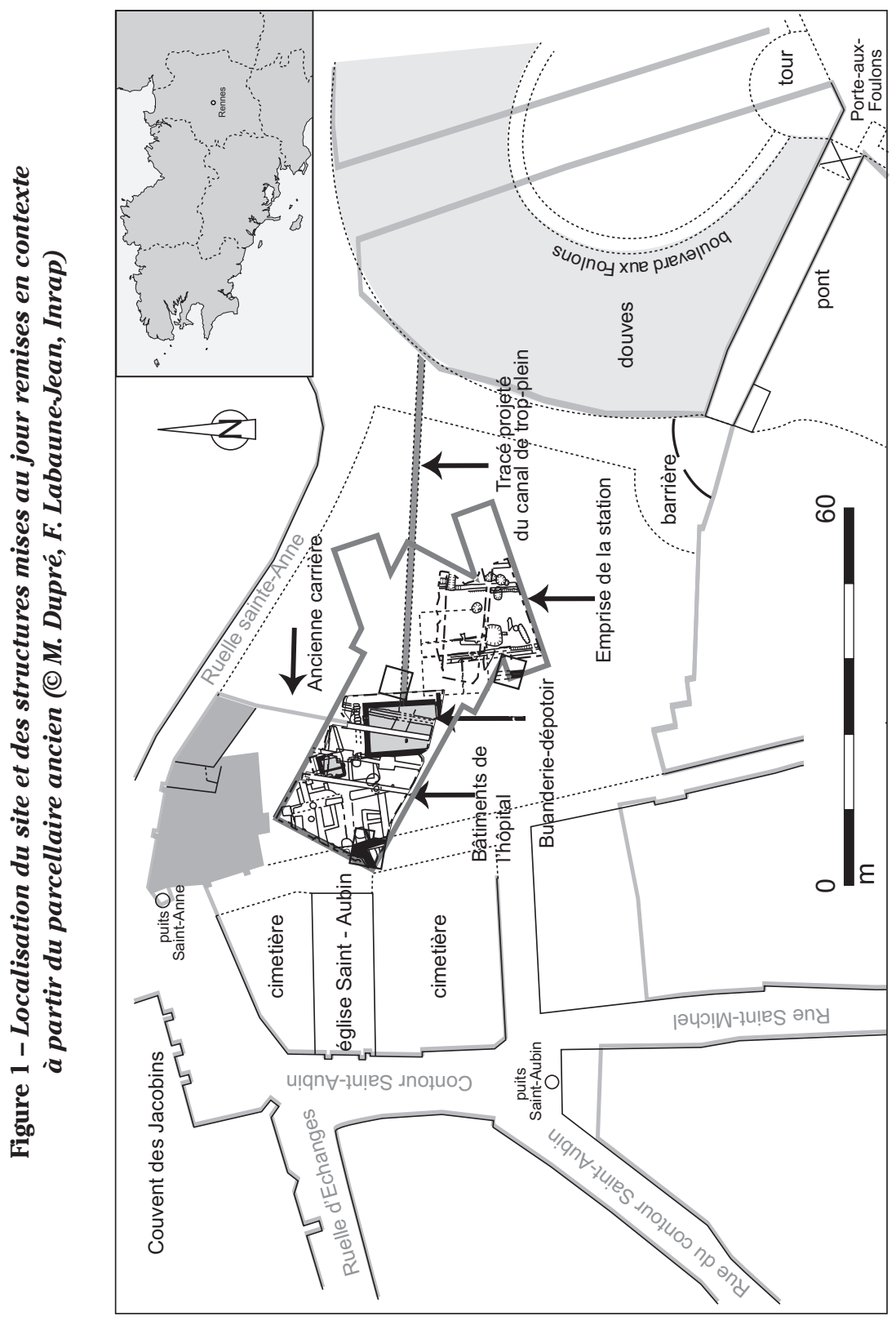




\section{État des connaissances sur les hôpitaux rennais}

Avant les données archéologiques, ce sont les sources documentaires qui renfermaient les principales informations sur les établissements de santé présents à Rennes. Comme le rappelle Jean-Charles Sournia ${ }^{5}$, la ville ne semble pas bénéficier durant le Moyen Âge d'une véritable politique de santé de la part des ducs de Bretagne.

Que ce soit au Moyen Âge ou au début de l'époque moderne, l'existence de ces institutions tient principalement à la volonté d'institutions religieuses ou privées d'offrir des structures d'accueil pour les plus pauvres et des hébergements temporaires pour les pèlerins. La notion sanitaire s'y greffe par voie de conséquence, mais les structures hospitalières de ces périodes ne correspondent pas aux bâtiments tels qu'on les conçoit de nos jours en tant que seuls centres de soins. L'hôpital est une œuvre de charité qui trouve son origine dans la pratique du devoir d'hospitalité. Quand on revient sur les définitions anciennes du terme, on y voit que " l'hospital" est " un lieu pieu et charitable où on reçoit les pauvres pour les soulager en leurs necessitez. " Les structures peuvent alors se classer en trois groupes suivant les activités dispensées : les établissements d'isolement pour gérer les pandémies en isolant les malades (léproseries, lazarets...), ceux de bienfaisance assurant des soins d'accompagnement (orphelinats, maisons de retraite) et les lieux d'hébergement (hospices) et d'enfermement. On peut alors les distinguer par leurs appellations : hôpital général (mendiants), hôtel-Dieu (tous les malades), Petites Maisons (fous), les Enfants rouges, de la Trinité ou du Saint-Esprit (orphelinats), Quinze-Vingts (aveugles), établissements de Saint-Jacques (pèlerins).

Les historiens de ce domaine montrent que l'essor des fondations se place entre le $\mathrm{XI}^{\mathrm{e}}$ et le $\mathrm{xIII}{ }^{\mathrm{e}}$ siècle, en accompagnement des fortes croissances urbaines et que la multiplication des établissements intervient entre les $\mathrm{XI}^{\mathrm{e}}$ et $\mathrm{XV}^{\mathrm{e}}$ siècles ${ }^{6}$.

Ces hôpitaux présentent des points communs caractéristiques : l'implantation à proximité d'un axe de communication (route ou voie d'eau), d'une chapelle, d'une ou plusieurs salles communes, d'un jardin et, dans certains cas, d'un cimetière. Celui de Sainte-Anne répond à tous ces critères : une installation en sortie de ville sur le grand axe routier ${ }^{7}$ menant au nord de la région rennaise, vers Saint-Malo et Dinan, avec des structures d'accueil et un cimetière. Ce dernier a pu être partiellement abordé lors de la fouille menée en fin d'année 2013, par une équipe Inrap, dans le cadre

5. SournIA, Jean-Charles, "Histoire des hôpitaux de Rennes ", Histoire des sciences médicales, $\mathrm{n}^{\circ} 7,1973$, p. 181-223. Nous renvoyons à cet auteur qui s'est penché de façon précise, dans cet article, sur la mise en place des différentes institutions médicales de Rennes jusqu'à la mise en place de l'hôpital actuel de Pontchaillou.

6. Cf. travaux de CABAL, Michel, Hôpitaux. Corps et âmes, éd. Rempart, 2001, 165 p. et IMBERT Jean, Les hôpitaux en France, Paris, PUF, coll. "Que sais-je? ", 1966, 128 p.

7. Axe qualifié en 1368 de "grand chemin rennais " (Arch. dép. d'Ille-et-Vilaine, 18H2, acte de fondation du couvent des jacobins, 23 avril 1368). 
de l'implantation de la seconde ligne de métro. Selon la classification des édifices médiévaux hospitaliers de Michel Cabal, l'établissement de SainteAnne entre dans le groupe de type accumulatif, avec des nefs accolées, greffées les unes aux autres selon l'espace disponible alloué.

\section{Les données d'archives}

Les recherches documentaires en vue de l'opération de fouilles ont été menées en amont et pendant la phase terrain par Nicolas Cozic ${ }^{8}$. Elles nous montrent qu'avant l'installation hospitalière, cet espace correspond à un tissu urbain complexe où se côtoient de la voirie, les "fossés à Gahier " ceinturant les faubourgs de Rennes à cet endroit, des constructions religieuses (église paroissiale primitive de Saint-Aubin et son cimetière), des habitats privés ainsi que des jardins avant que n'apparaisse, à la fin du $\mathrm{XVI}^{\mathrm{e}}$ siècle, la partie orientale de l'actuelle place Sainte-Anne (ancienne place du cimetière Sainte-Anne). Les données sur l'hôpital restent toutefois relativement maigres (trois liasses aux Archives municipales de Rennes) du fait de la durée de vie limitée de l'établissement et de son annexion à l'hôpital Saint-Yves dans la seconde moitié du Xvie siècle, pendant une dizaine d'années, avant qu'il ne cesse complètement de fonctionner. Les papiers le concernant ont donc été, au mieux, réunis à ceux de Saint-Yves ${ }^{9}$ ou plus souvent perdus.

L'étude a toutefois permis d'appréhender trois phases chronologiques sur cet espace : 1) de 1340, date de la fondation, à la fin du Xve siècle : mise en place de l'hôpital; 2) de la fin du Xve aux environs de 1564 : son essor, son déclin puis l'arrêt de l'activité hospitalière; 3) enfin, de 1564 jusqu'au $\mathrm{XIX}^{\mathrm{e}}$ siècle : réorganisation de l'espace et mise en place de la place.

La création de l'établissement de Sainte-Anne s'intègre dans un phénomène de laïcisation de l'assistance manifesté par les élites bourgeoises à partir de la fin du XIII ${ }^{\mathrm{e}}$ siècle. Ici, sa mise en œuvre est due à l'initiative de l'abbé et de l'aumônier du monastère de Saint-Melaine, de quelques bourgeois et de dix confréries de métier ${ }^{10}$. Elle est liée à l'augmentation de la population et aux crises démographiques des premières décennies du $\mathrm{XIV}^{\mathrm{e}}$ siècle. Parmi ces notables, Jean Raberge et Perrot Chouan, possesseurs de deux propriétés contiguës près du cimetière Saint-Aubin, sont chargés, comme procureurs, de mener à bien cette construction.

Les documents disponibles indiquent un espace enclos dans un mur délimitant le " pourprins Sainte-Anne " avec, à l'est, l'espace cultivé de

8. CozIC, Nicolas, "Étude des archives ", dans PoullLe, Dominique, " Métro-Val, station Place Sainte-Anne ", Rapport de fouilles de sauvetage, Rennes, 1998-1999, inédit.

9. Ces documents figurent aux Archives départementales d'Ille-et-Vilaine (H dépôt Rennes, $\mathrm{n}^{\circ} 1$ à 550).

10. Arch. mun. de Rennes, liasse 327. Acte de fondation de l'hôpital Sainte-Anne, décembre 1340 . 
jardins et à l'ouest, auprès d'une chapelle hospitalière antérieure à 1455, le (ou les) bâtiment(s) sur une surface de sept mètres par vingt et un mètres ${ }^{11}$.

En 1460, une supplique du gardien de Sainte-Anne réclame à la ville une aide pour aider à la réparation des " mesons d'icelui hospital qui sont tous en ruine ". Les archives mentionnent bien des financements accordés en 1462 pour des travaux de reconstruction ainsi que la réalisation d'un pavage, l'année suivante, le long de l'établissement. Les campagnes de travaux continuent en 1468, ainsi que pour l'hôpital Saint-Yves, résultant d'une volonté des autorités urbaines d'agrandir les deux établissements pour offrir des structures adaptées à l'accroissement de la population urbaine de l'époque. Cette augmentation des capacités hospitalières s'avère d'actualité car des vagues d'épidémies et le conflit franco-breton sévissent.

\section{Un important dépotoir}

Parmi les vestiges fouillés en 1998-1999, la vaste cuve d'une buanderie, utilisée comme dépotoir avant son remblaiement définitif au milieu du $\mathrm{XVI}^{\mathrm{e}}$ siècle, est sans doute mise en place lors de ces étapes d'aménagement complémentaire. Installée dans le creusement d'une ancienne carrière médiévale, elle se compose d'un vaste espace rectangulaire de maçonnerie délimitant la réserve d'eau d'une contenance d'au moins $400 \mathrm{~m}^{3}$. Restée inachevée pour une raison inconnue, la cuve a été transformée en dépotoir, fournissant ainsi une extraordinaire quantité de matériel archéologique. Outre deux tonnes de récipients en céramique, on compte bon nombre de verres, d'écuelles et peignes en bois, de jeux de marelles gravées sur des ardoises, de déchets alimentaires, de fragments d'étoffes, etc., autant d'objets illustrant la vie quotidienne du secteur. L'ensemble présente un profil très homogène datable de la première moitié du XvI ${ }^{\mathrm{e}}$ siècle ${ }^{12}$.

11. "[...] des habitations qui seront faites pour ceux qui seront reçus audit hôpital ", Jones, Michael, Recueil des actes de Jean IV, duc de Bretagne, Paris, Klincksieck, p. 704, $\mathrm{n}^{\circ} 134$, fondation du 8 février 1369 .

12. Le mobilier céramique et les objets en verre ont fait l'objet de plusieurs articles; LABAUNE-JEAN, Françoise, "Le vaisselier rennais du XV au XVII ${ }^{\mathrm{e}}$ siècle ", dans DiETRICH, Anne, RAVoIRE, Fabienne (dir.), La cuisine et la table dans la France de la fin du Moyen Âge, Actes du congrès de Sens (janvier 2004), Caen, Publications du Crahm, 2008, p. 411-424; LABAunEJEAn, Françoise, "Rennes, place Sainte-Anne. Aperçu du mobilier de l'hôpital ", dans LE Clech-Charton, Sylvie (dir.), Les établissements hospitaliers en France du Moyen Âge au $X I X^{e}$ siècle, Actes du colloque Espaces, objets, populations dans les établissements hospitaliers du Moyen Âge au XIX siècle, Tonnerre 4 et 5 septembre 2008, Dijon, Éditions universitaires, 2010, p. 255-266 ; LABAUNE-JEAN, Françoise, « Le verre soufflé-moulé à partir de trois lots en contexte hospitalier, à Rennes, place Sainte-Anne (Ille-et-Vilaine, France) ", dans FonTAINEHodiamont, Chantal (dir.), D'Ennion au Val Saint-Lambert. Le verre soufflé-moulé. Actes des $23^{e}$ Rencontres de l'Association française pour l'Archéologie du Verre, octobre 2008 (Scientia Artis, 5), Bruxelles, Institut royal du Patrimoine artistique, Scientia Artis, 2010, p. 391-396; Labaune-Jean, Françoise, PoullLé, Dominique, " Un dépotoir en contexte hospitalier à Rennes, place Sainte-Anne. Aperçu du vaisselier en usage au début XVI ${ }^{\mathrm{e}}$ siècle ", dans $\grave{A}$ propo $(t) s$ de l'usage, de la production et de la circulation des terres cuites dans l'Europe du Nord-Ouest (XIV-XVte siècle), Actes de la table-ronde, Caen, décembre 2007. Caen, 


\section{Les traces d'un artisanat méconnu}

C'est dans ce contexte qu'ont été découverts plusieurs éléments en plomb témoignant des croyances et de la ferveur des personnes accueillies dans cet ensemble hospitalier. Plusieurs moules en schiste gravé témoignent de l'artisanat du plomb. Ils sont tous réalisés à partir de petites plaques de grès armoricain au grain très fin. Cette finesse de texture permet, après un polissage soigneux, d'obtenir une surface de travail parfaitement plane, ainsi qu'une grande précision dans la gravure de la pièce à couler. La nature de la pierre est directement choisie en fonction de l'usage souhaité. Elle doit, entre autres, répondre à des critères d'abrasion et de résistance aux chocs thermiques. Ces moules sont, en cela, tout à fait similaires à des moules trouvés en contexte d'ateliers lors d'une intervention récente au Mont-Saint-Michel ${ }^{13}$.

\section{La réalisation des pièces}

L'artisanat du plomb repose sur un principe technique commun au travail des autres métaux. L'artisan dispose de valves de moule gravées en creux, qu'il associe de manière à couler le métal en fusion dans le négatif de l'objet ainsi constitué. Les moules découverts sont de forme rectangulaire ou plus souvent trapézoïdale. Revenant régulièrement, cette forme semble correspondre à un besoin particulier, inconnu pour le moment. Les objets à fondre fonctionnent ici selon un système classique nécessitant la mise en œuvre de deux valves en miroir. La complexité des objets peut, quelquefois, nécessiter des agencements plus importants de quatre à cinq pièces mâles et femelles. Les valves sont dotées de perforations traversantes et d'aménagements coniques creux. Une fois les deux pièces assemblées, du plomb est coulé dans les perforations et vient occuper le cône. Cette technique sert au maintien des deux pièces le temps de la coulée et du refroidissement, ainsi qu'à l'ajustement précis pour éviter les décalages. Celle-ci s'effectue au moyen d'un cône poursuivi par un canal de section biseautée, les deux

\footnotetext{
Publications du Crahm, 2011, p. 231-244; Labaune-JEAn, Françoise, Notices : « Du baume au cœur ", "Pustules, bubons... et coups de soleil " et " Mange ta soupe et guéris vite! " dans Menez, Yves, BerRetrot, Françoise, Veneau, Émilie, Herry, Nolwenn, Soyons fouilles. Découvertes archéologiques en Bretagne, (Journal d'exposition, Château de la Roche-Jagu avril - octobre 2011), La Roche-Jagu, CG 22, 2011, p. 48-49.

13. Menée sur une campagne d'hiver 2004-2005, cette fouille conduite par l'Inrap a livré un important lot de plus de 200 moules dont la publication est en cours. Une première présentation a été menée dans le cadre du colloque de Cerisy-la-Salle en 2008, consacré à l'iconographie du Mont-Saint-Michel et de l'archange. BRUNA, Denis, LABAUNEJEAN, Françoise, "Images de l'archange saint Michel dans les moules à enseignes de pèlerinage récemment découverts au Mont-Saint-Michel ", dans BouET, Pierre, OtRANTO, Giorgio, VAuchez, André, VInCENT, Catherine (dir.), Représentations du Mont et de l'Archange saint Michel dans la littérature et dans les arts (Rappresentazioni del Monte e dell'Archangelo san Michele nella letteratura e nelle arti), Bari, Éditions Edipuglia, 2011, p. 183-197. LaBAune-Jean, Françoise, « Une production d'enseignes de pèlerins au Mont Saint-Michel ", Archéopage, $n^{\circ}$ 18, Migrations, Paris, 2007, p. 80-81.
} 
étant aménagés à partir du côté le plus étroit du moule. Les expérimentations archéologiques montrent que ces moules étaient chauffés de manière à éviter les chocs thermiques et la prise trop rapide du métal en fusion. De taille petite à moyenne (longueur maximale comprise entre 5 et $10 \mathrm{~cm}$ pour une épaisseur inférieure à $1,5 \mathrm{~cm}$ ), les plaques sont gravées en faible creux, de l'ordre de quelques millimètres. C'est l'une des spécificités de cet artisanat, reposant sur la production de fines feuilles de métal ajourées à usage varié. Les valves découvertes ici servent toutes à la production d'un seul objet par moule.

\section{Des moules pour quelle production?}

Les sept valves de la place Sainte-Anne correspondent à différents objets plus ou moins identifiables (figure 2). Les deux premiers permettent la réalisation de rouelles à huit perles. La large perforation circulaire placée au centre de la rouelle sert également à l'évacuation du surplus de métal. Le troisième moule présente une ébauche assez grossière en forme de crucifix. L'irrégularité de son tracé évoque plus un travail de débutant qu'une œuvre aboutie; la surface de la croix et une partie des extérieurs sont recouvertes de graffiti géométriques. Le moule suivant est gravé d'une pièce assez énigmatique en forme de "raquette " aplatie dont la surface est entièrement décorée de motifs losangiques; le centre est, quant à lui, orné d'une rosace à huit branches emboîtées. L'usage d'un tel objet, une fois fondu, reste actuellement sans comparaison. Le dernier élément présente un motif en creux permettant de mouler des cercles assez larges en anneau bombé. Là encore, la destination finale reste à définir; l'usage du calcaire pour cette pièce permet d'y voir un contre-moule; il reste un trou à l'emplacement de la pointe du compas utilisé pour tracer la forme circulaire. L'ensemble est enfin complété par deux petits éclats de moules et deux pièces inachevées. La première de forme trapézoïdale à la surface soigneusement polie a été abandonnée avant la gravure; il en va de même pour la seconde, qui a été recouverte de graffiti sur l'ensemble de ses faces.

\section{Un objet énigmatique}

Taillé dans le même type de schiste, il faut aussi signaler un fragment orné, sur toutes ses faces, de gravures difficiles à interpréter. Ce schiste ardoisier à grain très fin est de forme rectangulaire $(1: 3,45 \mathrm{~cm}$; ép. : $2,7 \mathrm{~cm}$; $\mathrm{L}: 8,75 \mathrm{~cm}$ ). Une de ses extrémités est cassée. Toutes les surfaces de ce parallélépipède ont été soigneusement polies avant d'être gravées. Les motifs profonds de $0,1 \mathrm{~cm}$ représentent des lettres, des idéogrammes, un blason et une rosette à six pétales incluse dans un cercle. La fonction de cet objet reste à ce jour énigmatique (figure 3). 
Figure 2 - Différentes valves de moule (ou essais avortés) découverts sur le site (@ M. Dupré, F. Labaune-Jean, Inrap)
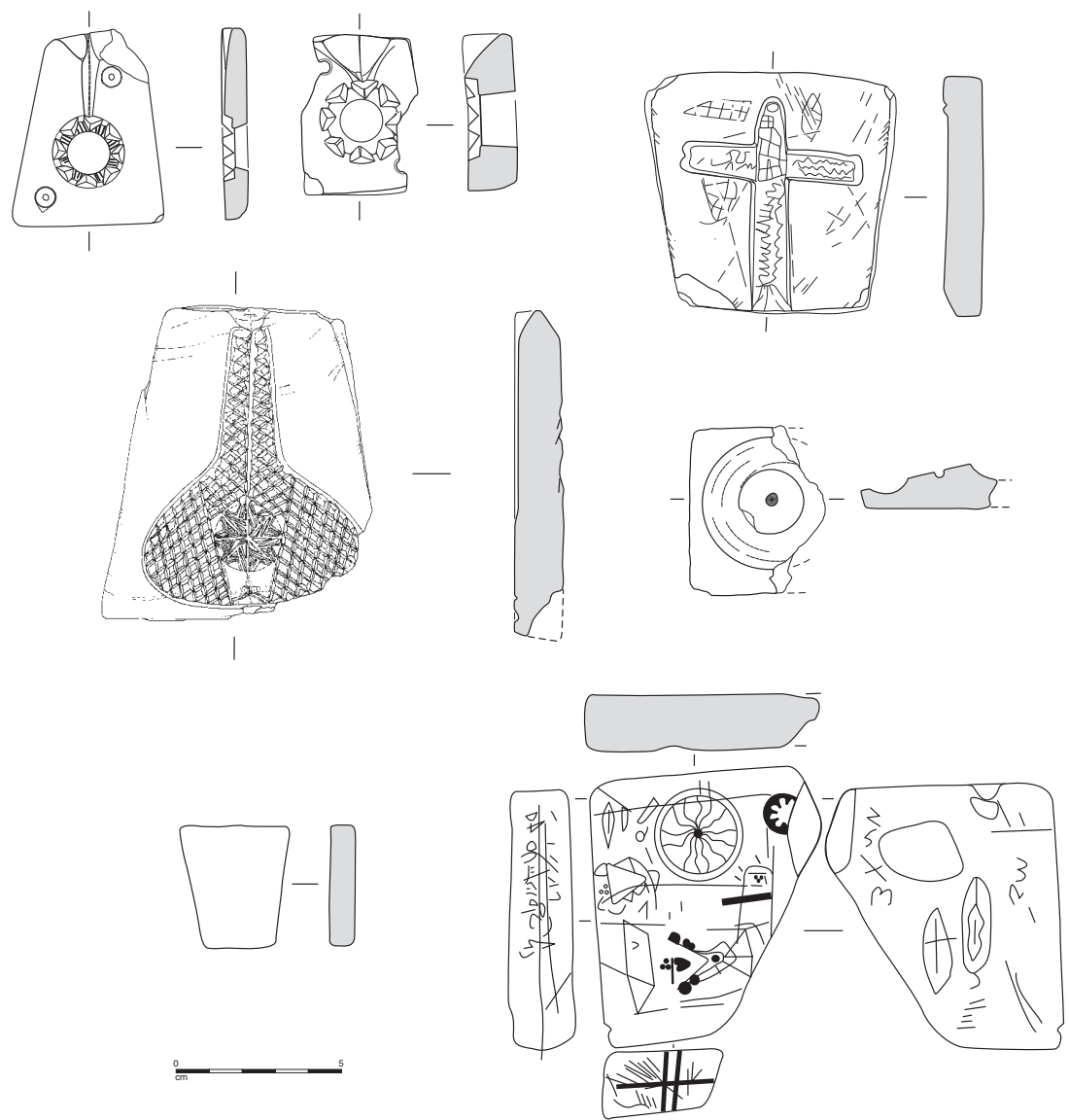

\section{Les productions associées}

Plusieurs objets en plomb en rapport avec ces moules ont été recueillis lors du tamisage des remblais. Si la première rouelle en plomb d'un diamètre externe de 1,93 cm est en mauvais état de conservation (forte corrosion de la pièce), la seconde est, quant à elle, intacte (1,95 cm de diamètre). Il s'agit toutefois d'un objet inachevé, car il n'a pas été ébarbé. Aucune des deux ne fonctionne avec les valves de moules mis au jour. L'usage de ces objets est toujours débattu ${ }^{14}$ (figure 4).

14. QuÉRÉ, Michel, «Étude de 88 rouelles tronconiques et d'une fusaïole provenant de la région de Saint-Genou (Indre) ", Bulletin du groupe d'histoire et d'archéologie de Buzançais, 
Figure 3 - Fragment de schiste gravé à usage énigmatique (C) M. Dupré, Inrap)
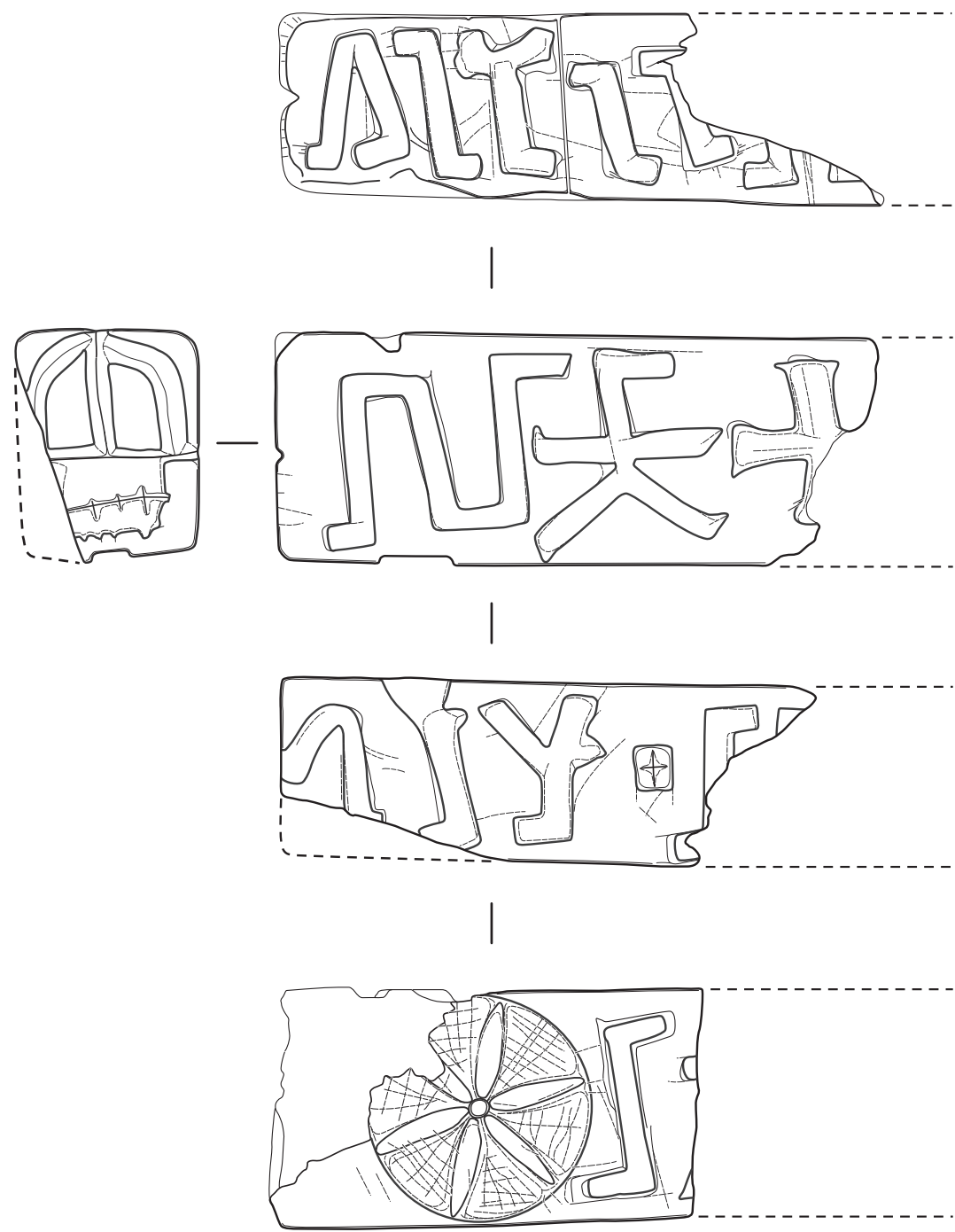

\section{$\mathrm{cm}$}

$\mathrm{n}^{\circ}$ 24, 1992, p. 31-52; QuERE, Michel, " Rouelles tronconiques provenant de la région de Saint-Genou (Indre) (2 $2^{\mathrm{e}}$ partie) ", Bulletin du groupe d'histoire et d'archéologie de Buzançais, $\mathrm{n}^{\circ} 27,1995$, p. 15-33. 


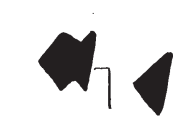

1
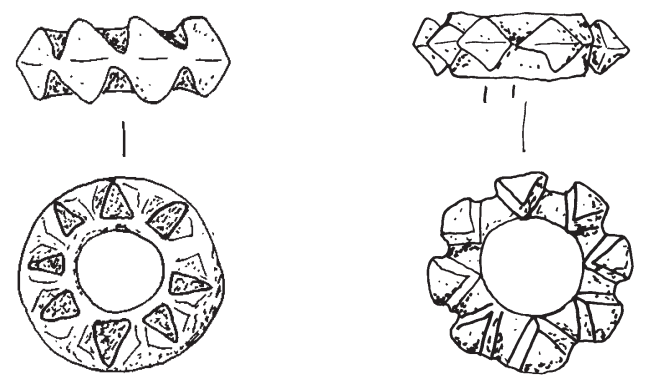

5

0

Figure 4 - Deux rouelles en plomb retrouvés dans le comblement du dépotoir (C F. Labaune-Jean, Inrap)

Toujours dans le même alliage plomb-étain, il faut signaler un petit crucifix moulé (figure 5). Fabriqué à partir d'un moule à deux valves de manière à constituer un pendentif recto-verso, il a fait l'objet d'un traitement particulier : sa surface est entièrement étamée ce qui lui donne un aspect argenté. La face montre un Christ en croix grossièrement esquissé, alors que le

Figure 5 - Petit crucifix à décor moulé en alliage étamé

() S. Jean, F. Labaune-Jean, Inrap)
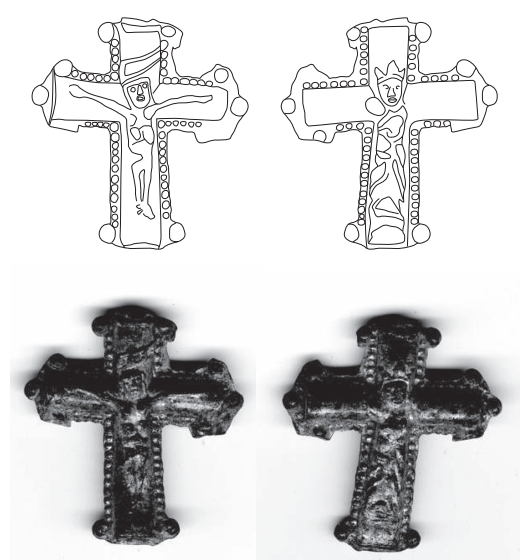

0

$\mathrm{cm}$ revers est orné d'une Vierge à l'Enfant (de la même main). Les bords soudés sont décorés de petites perles accolées et de trois perles plus grosses, disposées aux angles et à l'extrémité des branches de la croix. Le système de suspension a disparu.

L'autre élément de bijouterie en plomb-étain est un petit pendentif circulaire à médaille (figure 6). Il est constitué d'un cerclage circulaire à anneau de suspension qui enserre deux petites médailles moulées en faible relief. La première montre le buste d'un évêque coiffé d'une mitre, avec ses attributs : la crosse et une clé; la seconde médaille montre un sujet plus classique de Vierge à l'Enfant. La réalisation est l'œuvre d'une même personne, comme le mon- 
trent les personnages esquissés à grandes lignes. Cette caractéristique et la présence d'une bande de petites perles sur la bande de soudure du cerclage, permettent d'y voir le travail du même artisan que pour le crucifix ${ }^{15}$.

\section{Les objets liés au pèlerinage}

Une enseigne intacte et les fragments d'une autre ont été découverts lors du tamisage du remblai comblant le dépotoir. Ces deux objets correspondent à des insignes - ou enseignes - de pèlerins. Ils ont l'apparence d'une broche que les fidèles arborent sur leurs vêtements au moyen d'une épingle; ils sont largement répandus dans la vie quotidienne et religieuse de la fin du Moyen Âge. Tels des souvenirs en mémoire du pèlerinage effectué, les insignes sont achetés auprès de " faiseurs d'enseignes " qui installent leurs étals près des églises. Les deux enseignes de Rennes, comme les quelques milliers que nous conservons aujourd'hui dans les collections publiques et privées ${ }^{16}$, sont réalisées dans un alliage de plomb et d'étain, matériau facile à travailler et bon marché. Cette dernière particularité permet à tous les groupes de la société d'acquérir ces pièces pour témoigner de leur attachement dévotionnel à un saint ou à un sanctuaire.

La première enseigne se compose d'une plaque en plomb, avec un décor ajouré en faible relief $(5,65 \mathrm{~cm}$ de hauteur; $2,65 \mathrm{~cm}$ de largeur maximale; 0,05 cm d'épaisseur) (figure 7); l'ardillon servant à la fixation est conservé à l'arrière. Le décor se décompose en deux registres. Dans la partie basse, on trouve deux personnages masculins, de face, portant de longues robes drapées et présentant leurs insignes; la tête d'un troisième personnage apparaît aux pieds de deux autres. La scène est incluse dans un cercle plat sur lequel apparaissent deux inscriptions en faible relief

15. Signalons la présence, dans les découvertes du site de la cour des écoles au MontSaint-Michel, d'une valve servant à la fabrication des cerclages de ce type de pendentif.

16. BRunA, Denis, "Enseignes de pèlerinage et enseignes profanes ". Catalogue des collections du Musée National des thermes de Cluny. Paris, Réunion des musées nationaux 1996, 383 p.; Bruna, Denis, Saints et diables au chapeau. Bijoux oubliés du Moyen Âge, Paris, Seuil, 2007. 
Figure 7 - Enseigne de pèlerinage à l'effigie de Saint Côme et Saint Damien (C) S. Jean, F. Labaune-Jean, Inrap)

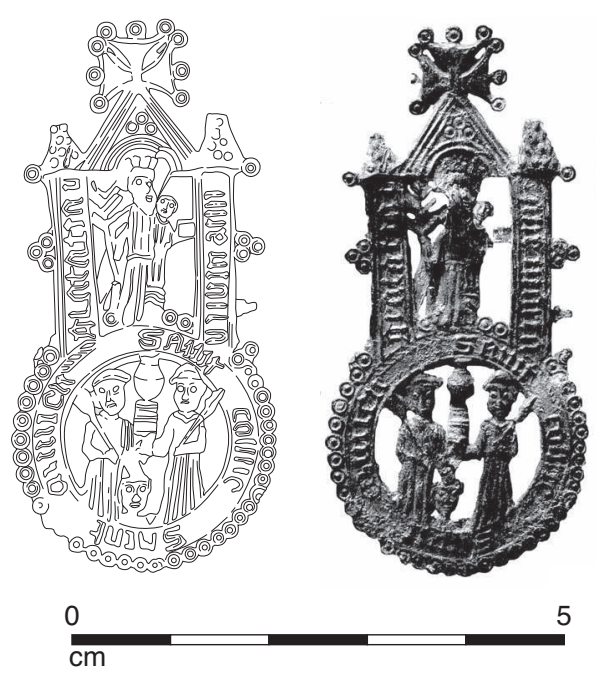

indiquant le nom des deux personnages figurés : saint Côme et saint Damien. Une ligne de doubles cercles pointés ceinture ce bandeau sur l'extérieur. La partie supérieure montre une figure de Marie portant le Christ enfant; elle se tient dans une niche architecturale décorée de perles et dont le fronton triangulaire se termine par les trois branches d'une croix perlée. Les deux piédroits portent une inscription en faible relief. Côme et Damien étaient des frères jumeaux qui pratiquaient gracieusement la médecine dans une ville de Cilicie, c'est pourquoi ils sont représentés sur l'enseigne avec des attributs médicaux : le bonnet et la robe de médecin, des instruments chirurgicaux et un pot à pharmacie.

La seconde plaque de plomb fragmentée est de forme rectangulaire (figure 8). Le décor reconstitué sur deux registres montre, en partie basse, les effigies de saint Jean-Baptiste et saint Sébastien, séparés par une Vierge à l'enfant. Le registre supérieur délimité par une architecture stylisée ne conserve qu'un personnage couronné, de petite taille, vêtu d'une longue tunique et présentant devant lui une sorte de coffret.

\section{Les connaissances antérieures}

Cette étude a également été l'occasion de reprendre les collections anciennes de la ville. En effet, ces deux enseignes de plomb ne sont pas les premiers insignes découverts à Rennes : au XIX ${ }^{\mathrm{e}}$ siècle, quelques pièces avaient été retirées de la Vilaine et publiées par l'archéologue Adolphe Toulmouche dans sa monumentale étude, Histoire archéologique de l'époque gallo-romaine de la ville de Rennes (1847). Excepté les objets associés au pèlerinage du Mont-Saint-Michel et à celui de Saint-Maur-des-Fossés (près de Paris), les autres n'avaient pas été identifiées par l'" antiquaire ". Les sanctuaires d'origine de ces enseignes ont pu enfin être retrouvés : SaintGilles-du-Gard et Saint-Mathurin-de-Larchant (figure 9).

Le moule mentionné et dessiné dans l'ouvrage d'Adophe Toulmouche ne figure plus dans l'inventaire des collections du musée de Bretagne 


\section{Figure 8 - Seconde enseigne fragmentaire mais dont l'agencement est restituable (C) S. Jean, F. Labaune-Jean, Inrap)}
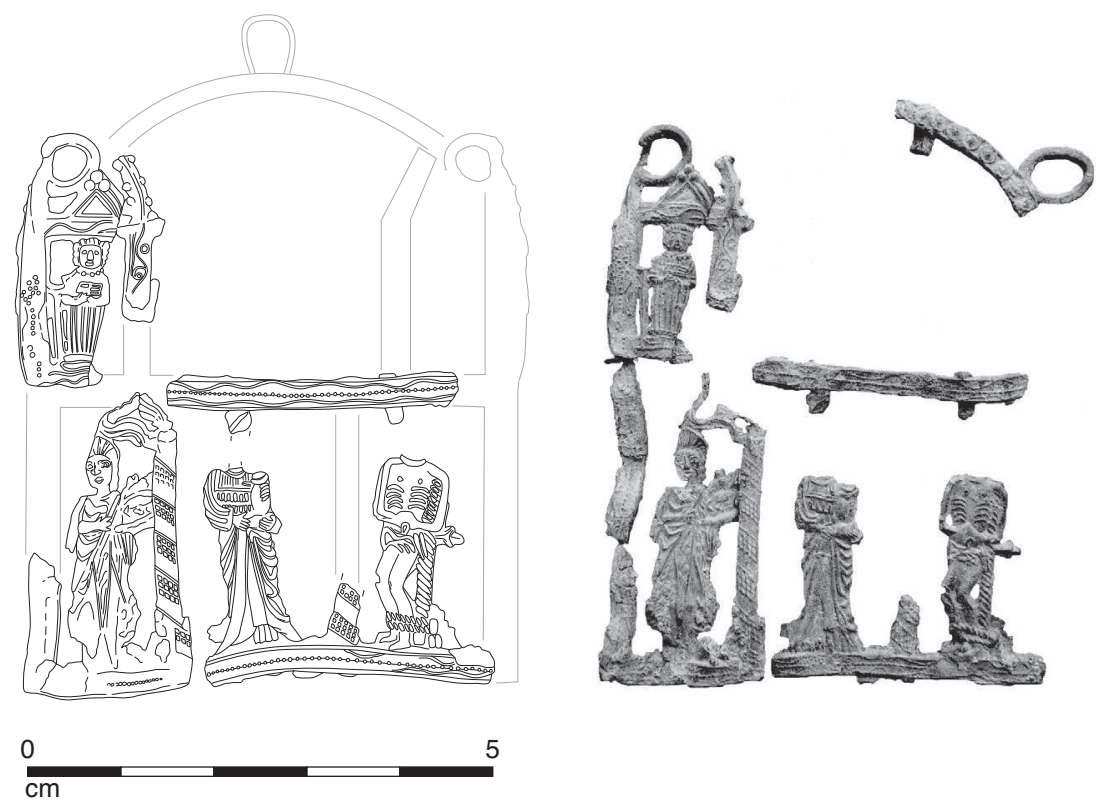

$\mathrm{cm}$

de $1876^{17}$. Grâce à ces relevés, on y observe une scène riche associant, sur un même plan, les différents actes de l'archange saint Michel : la pesée des âmes et le combat du diable. Le tout compose une enseigne en forme d'écu à décor supérieur de fleurons et de têtes. Il est daté du $\mathrm{XIV}^{\mathrm{e}}$ siècle par Adophe Toulmouche mais la similitude de composition avec deux moules découverts récemment au Mont-Saint-Michel, ferait pencher plutôt vers une datation plus proche de la fin du XIV ${ }^{\mathrm{e}}$ ou du début du $\mathrm{XV}^{\mathrm{e}}$ siècle $^{18}$ (figure 10).

On signalera également la mention en 1888, par Lucien Decombe, d'un moule en schiste ardoisier gravé (d'un Judas entraîné aux enfers?), trouvé lors de travaux dans des terrains de la rue Rallier, ancienne porte SaintMichel ${ }^{19}$. Envoyé à l'époque pour étude à la société des Antiquaires de

17. ANDRÉ, Auguste, Catalogue raisonné du musée d'archéologie et de céramique et du musée lapidaire de la ville de Rennes, Seconde édition, revue et augmentée, Rennes, Imp. Leroy fils, 1876.

18. Dans un même ordre d'idée, Adolphe Toulmouche mentionne également un autre moule servant à la fabrication de grelots, vraisemblablement comparable aux exemplaires récents de la fouille de la cour des écoles au Mont-Saint-Michel, Toulmouche Adolphe, Histoire archéologique de l'époque gallo-romaine de la ville de Rennes, Rennes, Deniel, 1847, p. 148; Bruna Denis, LaBAunE-JEAn, Françoise, op. cit., p. 183-197.

19. Decombe, Lucien, "Compte rendu de séance. Exhibitions. ", Bulletin de la société archéologique d'Ille-et-Vilaine, tome XVIII, Rennes, 1888, p. VI-VII. 
Figure 9 - Valve de moule et enseignes de pèlerinage retrouvés dans les travaux de dragage de la Vilaine au XIX siècle (C) d'après A. Toulmouche 1847)

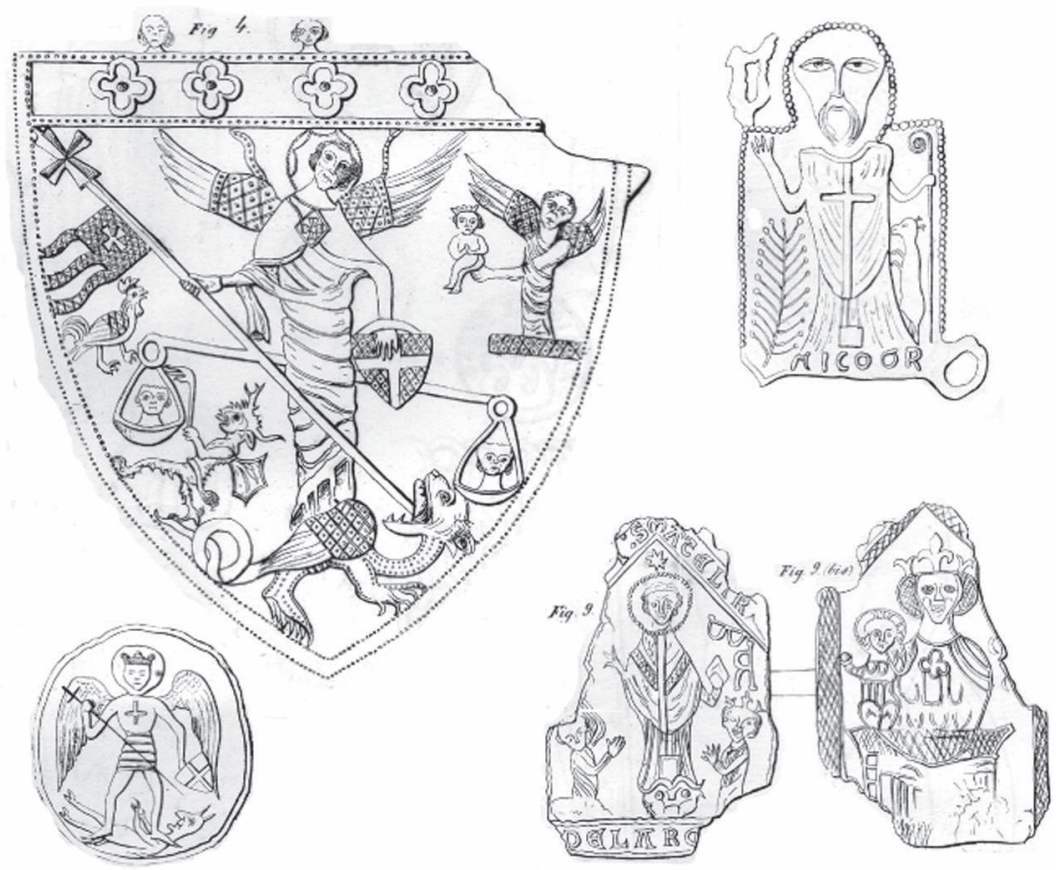

France, il ne figure plus dans les collections rennaises, mais il y a de fortes raisons de penser qu'il s'agit là encore d'un moule d'enseigne.

\section{L'artisanat du plomb étain}

Avec ces découvertes, le site livre de nouvelles informations sur un artisanat plutôt méconnu, le matériau peu prestigieux employé n'ayant pas attiré les érudits; il souffre également d'une pratique importante du recyclage entraînant la perte des pièces inutilisées ou des ratés de fabrication, plus simples à refondre qu'à transformer. Les structures artisanales mises au jour dans les ateliers découverts au Mont-Saint-Michel en 2004-2005 ${ }^{20}$ nous montrent que la mise en œuvre de cette activité ne laisse que peu de traces. En plus du recyclage, les structures de chauffe peuvent se dérouler sous un petit appentis, le mélange plomb-étain ayant un faible point

20. Labaune-Jean, Françoise, Le plomb et la pierre. Petits objets pour les pèlerins du MontSaint-Michel (14e-15e siècles), Caen, Publications du Cramh, à paraître. 
Figure 10 - Valves de moule à l'effigie de Saint Michel découverts au Mont-Saint-Michel lors d'une intervention archéologique Inrap en 20042005 (ㄷ H. Paitier, F. Labaune-Jean, Inrap)

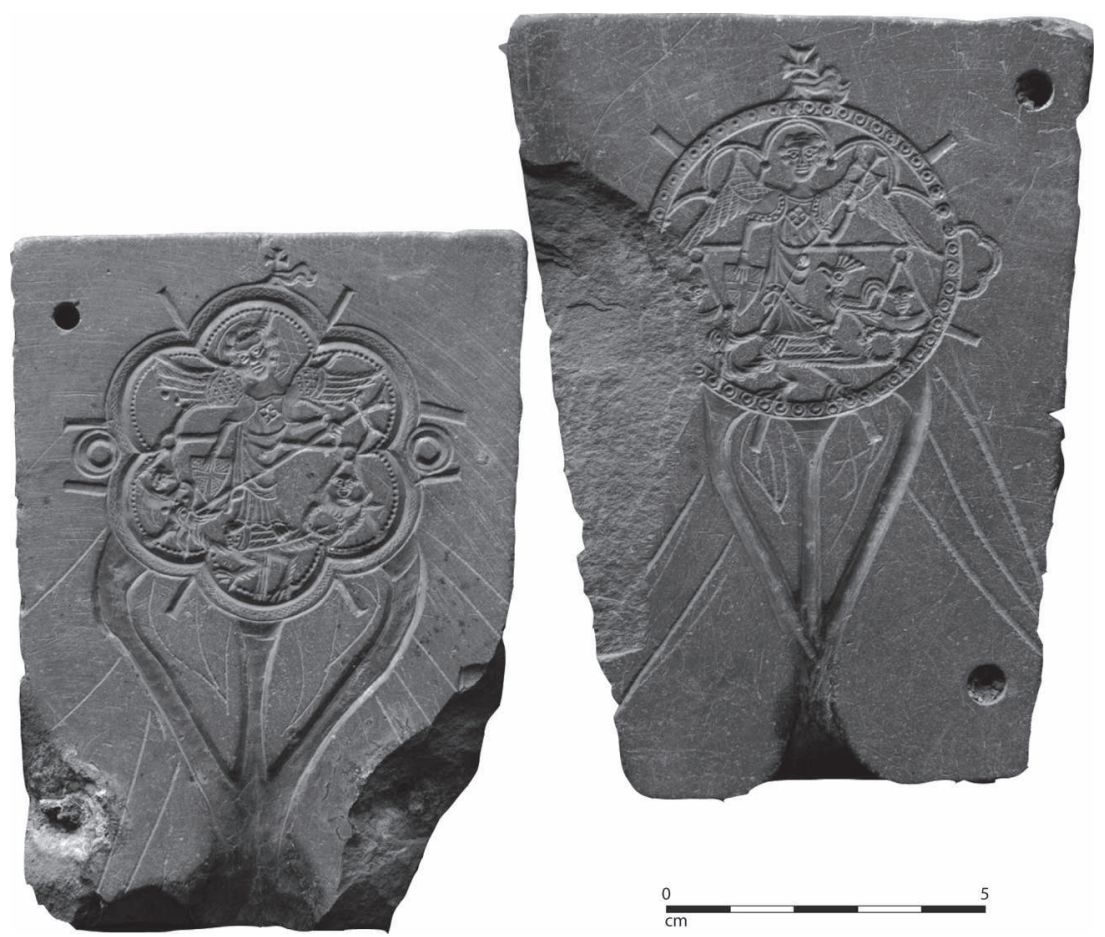

de fusion. En dehors de quelques assemblages pour les objets réalisés en plusieurs coulées, les moules montois nous montrent que c'est l'économie de gestes qui est le plus souvent recherchée par les artisans. Ainsi, pour les enseignes, la plaque décorée et ses anneaux ou son épingle de fixation sont coulés en même temps au moyen d'un assemblage de trois valves, une gravée de la partie figurée et au revers, l'aménagement de l'épingle sur la tranche des deux autres. Une fois coulée et refroidie, la pièce peut passer directement sur l'étal du marchand, permettant ainsi de produire et d'écouler une large variété de produits, tant de piété que d'usage quotidien. Cet artisanat par sa mise en œuvre plutôt aisée peut répondre à une large gamme de besoins, compensant le faible prix de vente par une adaptation à la demande et une diffusion souvent en grande quantité.

Si la présence des objets en plomb, pendentifs, enseignes et rouelles dans le comblement de ce dépotoir peut facilement s'expliquer, celle des moules demeure plus énigmatique. Même si la mise en œuvre de la fabri- 
cation d'objets en plomb ne réclame pas d'infrastructures importantes, leur existence n'est bien évidemment pas suffisante pour témoigner de cet artisanat à Rennes et dans le secteur de la place Sainte-Anne, même si cette hypothèse est à envisager. On peut également imaginer la présence, parmi les malades de l'hôpital, d'un artisan graveur continuant son activité dans l'attente d'une guérison prochaine, à moins que sa présence ne soit plus mercantile avec une installation à proximité d'une clientèle potentielle. N'oublions pas non plus l'afflux de pèlerins dans ce secteur de Rennes, le culte de Notre-Dame-de-Bonne-Nouvelle se déroulant juste à proximité dans le couvent des jacobins ${ }^{21}$. Avec l'enseigne à l'effigie de saint Côme et saint Damien, vénérés pour leur pratique de la médecine et leurs guérisons miraculeuses, et celle de saint Sébastien enrayant les épidémies de peste, on peut aussi envisager un artisan lié à l'hôpital ou profitant de sa proximité comme opportunité pour écouler sa production. Quelle que soit la solution retenue, ces pièces n'en demeurent pas moins importantes. Elles sont très bien datées du premier tiers du XVI ${ }^{\mathrm{e}}$ siècle par leur association à des monnaies et des verres. Illustrant la vie quotidienne et les croyances de l'époque, tous ces souvenirs témoignent aussi de l'importance des pèlerinages à l'époque médiévale et du fort désir de rapporter une part de sacré lors de ces si populaires mouvements de piété.

\section{RÉSUMÉ}

En 1998, le tracé de la première ligne de métro de Rennes entrainait une importante fouille archéologique sur l'actuelle place Sainte-Anne. L'occupation médiévale de ce secteur correspond à l'emplacement d'un hôpital urbain, créé au début du XIve siècle. La richesse des structures en mobilier a été l'occasion de se pencher sur les objets en usage à Rennes, dans ce type de contexte particulier. Cet article porte plus particulièrement sur l'artisanat et la production de petits objets en plomb, souvent mis de côté, car touchant l'art populaire.

\section{ABSTRACT}

In 1998, the route of the first underground line in Rennes resulted in an important archaeological excavation on Sainte-Anne square. In the Middle Ages, this area was the location of an urban hospital, created at the beginning of the fourteenth century. The wealth of the furniture structures offered an opportunity to look at the objects used in Rennes in this context. This article looks in particular at the crafts and the production of small lead objects, often set aside as belonging to popular culture.

21. Signalons que la fouille qui, menée par une équipe de l'Inrap dirigée par Gaëtan Le Cloirec, vient juste de se dérouler (fin 2011-2013) sur cet emplacement, a livré un nouveau moule en schiste trouvée dans une des sépultures mises au jour, pièce destinée à la fabrication de deux petits pendentifs losangiques à thème religieux (information inédite). 